KOMPONISTEN LEXIKON 


\section{KOMPONISTEN LEXIKON}

350 werkgeschichtliche Portraits

Zweite, überarbeitete und erweiterte Auflage

Herausgegeben von Horst Weber

J.B. Metzler · Bärenreiter 


\title{
Inhaltsverzeichnis
}

\author{
Vorwort $\mathrm{V}$ \\ Hinweise zur Benutzung VI \\ Verzeichnis der Abkürzungen und Siglen VII \\ Komponisten von A-Z $\quad 1-712$ \\ Verzeichnis der Autorinnen und Autoren 713
}

Redaktion: Gordon Kampe, Adrian Kuhl, Jana Zwetzschke

Gemeinschaftsausgabe der Verlage J.B. Metzler, Stuttgart und Weimar und Bärenreiter, Kassel

Bibliografische Information Der Deutschen Bibliothek

Die Deutsche Bibliothek verzeichnet diese Publikation in der Deutschen Nationalbibliografie;

detaillierte bibliografische Daten sind im Internet über $>$ http://dnb.ddb.de` abrufbar.

ISBN 978-3-476-01966-0

ISBN 978-3-476-05274-2 (eBook)

DOI $10.1007 / 978-3-476-052742$

Dieses Werk einschließlich aller seiner Teile ist urheberrechtlich geschützt. Jede Verwertung außerhalb der engen Grenzen des Urheberrechtsgesetzes ist ohne Zustimmung des Verlages unzulässig und strafbar. Das gilt insbesondere für Vervielfältigungen, Ubersetzungen, Mikroverfilmungen und die Einspeicherung und Verarbeitung in elektronischen Systemen.

(C) 2003 Springer-Verlag GmbH Deutschland

Ursprünglich erschienen bei J. B. Metzlersche Verlagsbuchhandlung

und Carl Ernst Poeschel Verlag GmbH in Stuttgart 2003

www.metzlerverlag.de

info@metzlerverlag.de 


\section{Vorwort}

Das Komponistenlexikon erscheint in neuer Gestalt und mit neuem Inhalt. Es ist schlanker, aber von gleichem Umfang. Die Welt der Musik und die Welt der Information haben sich in den vergangenen zwölf Jahren seit Erscheinen der ersten Auflage dramatisch verändert. Eine neue Generation von Komponisten ist nachgewachsen, Osteuropa nimmt intensiver als zuvor am europäischen Konzert teil und das Repertoire, das live und medial Verbreitung findet, ist breiter, auch diffuser geworden. Auf diese Entwicklungen galt es zu reagieren.

Für die zweite Auflage wurden 60 Artikel neu geschrieben, davon 40 als Neuaufnahmen; alle übrigen Artikel wurden von den Autoren oder der Redakion aktualisiert, die Literaturhinweise auf den Stand von 2003 gebracht.

Artikel und Literatur sind eine Auswahl. Ihre Kriterien sind dieselben geblieben wie in der ersten Auflage; Kontingente für Epochen, Stile und Nationen bilden das Gerüst, das notwendig subjektive Entscheidungen stützt. Ein deutlicher Akzent auf der Musik des 20. Jahrhunderts ist bewahrt.
Das Konzept, über das Komponieren der Komponisten zu informieren, erscheint im Zeitalter des Internet, das Daten im Überfluß zur Verfügung stellt, notwendiger denn je. Und die Begrenzung des Mediums Buch begründet seine Qualität. Denn die Notwendigkeit der Auswahl stiftet Orientierung.

Der Dank des Herausgebers gilt zuallererst seinen Autoren, vor allem denjenigen, die neue Beiträge für die zweite Auflage geschrieben haben, sodann dem Verlag, in dessen Händen Datenerfassung, Layout und Satzerstellung lag. Der Herausgeber dankt herzlich den Mitarbeitern, die an der Datenerfassung, Korrektur und Aktualisierung maßgeblichen Anteil hatten, Adrian Kuhl (Heidelberg), Gordon Kampe und Jana Zwetzschke (beide Essen). Sein besonderer Dank gilt Uwe Schweikert, der mit diesem Lexikon sein letztes Musikbuch als Lektor betreut hat und dem alle, die Bücher über Musik zu schätzen wissen, viel verdanken. Gerade für ihn wünsche ich mir, daß dieses Buch in der Flut medialer Informationen vielen Orientierung sein möge.

Essen, 26. September $2003 \quad$ Horst Weber 


\section{Hinweise zur Benutzung}

Im Titelkopf ist der erste Vorname der Rufname, sofern er nicht in Klammern steht. Im Text werden Komponisten, die in diesem Lexikon mit einem Artikel vertreten sind, nur mit Familiennamen genannt, andere Personen bei der ersten Nennung mit vollem Vor- und Zunamen. Auf unterschiedliche Schreibweisen von Nachnamen (Transliteration) wird verwiesen. Namen und Titel, denen die kyrillische Schrift zugrunde liegt, werden nach den Regeln der International Standard Organisation transliteriert, es sei denn der Komponist hat im Ausland eine Transkription autorisiert (z. B. Stravinsky); Lebensdaten sind bis 1917 nach dem Julianischen Kalender angegeben, die Datierung nach Gregorianischem Kalender steht in Klammern.

Einige Komponistennamen sind, dem Brauch in der Mediaevistik folgend, aber abweichend von anderen Musiklexika, unter ihrem Hauptnamen dem späteren Vornamen - eingeordnet (z. B. Guillaume de Machaut).

Werke des Komponisten, der im jeweiligen Artikel besprochen wird, erscheinen kursiv, alle anderen Werke in Anführungszeichen. Die Entstehung eines Werkes über mehrere Jahre wird durch Bindestrich angezeigt (z. B. 1788-89), verschiedene Fassungen sind durch Schrägstrich getrennt (1788/1789). Bühnenwerken sind die Übersetzung eines fremdsprachigen Originaltitels, der Namen des Librettisten sowie Ort und Jahr der Uraufführung in Klammern angefügt; die Enstehungszeit wird zusätzlich nur erwähnt, wenn bis zur Uraufführung ein größerer Zeitraum verstrichen ist.

Zitate werden in der Regel durch Kurztitel nachgewiesen, die bibliographischen Angaben sind in den Literaturhinweisen am Ende des Artikels komplettiert.

Die Literaturhinweise sind eine Auswahl. Sie enthalten keine Verweise auf einschlägige Lexika und Enzyklopädien wie "Die Musik in Geschichte und Gegenwart «, neubearbeitete, zweite Auflage herausgegeben. von Ludwig Finscher, Kassel und Stuttgart 1994ff., „The New Grove Dictionary of Music and Musicians«, Second edition edited by Stanley Sadie (u. a.) London 2001 oder umfangreiche Speziallexika wie "Pipers Enzyklopädie des Musiktheaters«, herausgegeben von Carl Dahlhaus $(\dagger)$ und dem Forschungsinstitut für Musiktheater der Universität Bayreuth unter Leitung von Sieghard Döhring, München 1986-1997 und „Komponisten der Gegenwart", herausgegeben von HannsWerner Heister und Walter-Wolfgang Sparrer, München 1992ff. Reihentitel sind nur in Ausnahmefällen genannt. 
Abkürzungen

$\begin{array}{llll}\text { Abh. } & \text { Abhandlung } & \text { Jb. } & \text { Jahrbuch } \\ \text { Ausg. } & \text { Ausgabe } & \text { Jh. } & \text { Jahrhundert } \\ \text { Bln. } & \text { Berlin } & \text { Kat. } & \text { Katalog } \\ \text { Bd., } & \text { Band } & \text { Kgr. } & \text { Kongreß } \\ \text { Bde. } & \text { Bände } & \text { Ldn. } & \text { London } \\ \text { Ber. } & \text { Bericht } & \text { Lpz. } & \text { Leipzig } \\ \text { Bibl. } & \text { Bibliographie } & \text { Mg. } & \text { Musikgeschichte } \\ \text { bzw. } & \text { beziehungsweise } & \text { Mn. } & \text { München } \\ \text { ca. } & \text { circa } & \text { mus. } & \text { musikalisch } \\ \text { Dépt. } & \text { Département } & \text { Mw. } & \text { Musikwissenschaft } \\ \text { Ders. } & \text { Derselbe } & \text { NA. } & \text { Neuausgabe } \\ \text { Dies. } & \text { Dieselbe(n) } & \text { Ndr. } & \text { Nachdruck } \\ \text { d.h. } & \text { das heißt } & \text { N.Y. } & \text { New York (City) } \\ \text { Diss. } & \text { Dissertation } & \text { o.J. } & \text { ohne Jahr } \\ \text { Dok. } & \text { Dokument } & \text { o.O. } & \text { ohne Ort } \\ \text { dt. } & \text { deutsch } & \text { op., } & \text { opus, opera } \\ \text { ebd. } & \text { ebenda } & \text { opp. } & \\ \text { engl. } & \text { Englisch } & \text { rev. } & \text { revidiert } \\ \text { erg. } & \text { ergänzt } & \text { s.o. } & \text { siehe oben } \\ \text { erw. } & \text { erweitert } & \text { s.u. } & \text { siehe unten } \\ \text { f.ff. } & \text { folgende } & \text { Stg. } & \text { Stuttgart } \\ \text { Faks. } & \text { Faksimile } & \text { u.a. } & \text { und andere, unter anderem } \\ \text { Ffm. } & \text { Frankfurt am Main } & \text { u.ö. } & \text { und öfter } \\ \text { fr. } & \text { französisch } & \text { u.U. } & \text { unter Umständen } \\ \text { Fs. } & \text { Festschrift } & \text { Univ. } & \text { Universität, University } \\ \text { Geb. } & \text { Geboren } & \text { usw. } & \text { und so weiter } \\ \text { gest. } & \text { gestorben } & \text { Veröff. } & \text { Veröffentlichung(en) } \\ \text { Hbg. } & \text { Hamburg } & \text { Verz. } & \text { Verzeichnis } \\ \text { Hdb. } & \text { Handbuch } & \text { vgl. } & \text { vergleiche } \\ \text { hrsg. } & \text { herausgegeben } & \text { WoO } & \text { Werk ohne Opuszahl } \\ \text { IGNM } & \text { Internationale Gesellschaft für Neue } & \text { WV } & \text { Werkverzeichnis } \\ & \text { Musik } & \text { z.B. } & \text { zum Beispiel } \\ \text { intern. } & \text { international } & \text { z.T. } & \text { zum Teil } \\ \text { IRCAM Institut de Recherche et Coordination } & \text { Zs. } & \text { Zeitschrift } \\ & \text { Acoustique/Musique } & \text { Ztg. } & \text { Zeitung } \\ \text { ital. } & \text { italienisch } & & \\ & & & \end{array}$


AfMw Archiv für Musikwissenschaft, 1 (1918-19) - 8 (1926), 9 (1952) ff.

BWV (Bach-WV) Wolfgang Schmieder, Thematisch-systematisches Verz. der Werke von Johann Sebastian Bach, Lpz. 1950,

2. überarbeitete Ausg. Wiesbaden 1990, kleine Ausg. hrsg. von Alfred Dürr und Yoshitake Kobayashi, ebd. 1998

BuxWV (Buxtehude-Werke-Verz.) Georg Karstädt, Thematisch-systematisches Verz. der musikalischen Werke von Dietrich Buxtehude, Wiesbaden 1974, 2. erw. und verbesserte Auflage 1985

CMM Corpus Mensurabilis musicae, Rom 1947-76, Neuhausen-Stg. 1977ff.

D (Deutsch-Verzeichnis) Otto Erich Deutsch, Schubert. Thematic Catalogue of All His Works in Chronological Order, Ldn. und N.Y. 1951, dt. NA Kassel 1978

DDT Denkmäler Deutscher Tonkunst, 1. Folge: 65 Bde., Lpz. 1892-1931, rev. Neuauflage Wiesbaden 1957-61 (fortgesetzt durch $\Rightarrow \mathrm{EDM}$ )

DTB Denkmäler der Tonkunst in Bayern. Denkmäler Deutscher Tonkunst, 2. Folge: 36 Bde., Lpz. 1900-23, Augsburg 1924-31, rev. Neuauflage Wiesbaden 1962ff.; Neue Folge Wiesbaden 1967ff.

DTO Denkmäler der Tonkunst in Osterreich, 83 Bde., Wien 1894-1938, Ndr. Graz 1959-60; Wien 1947ff.

EDM Das Erbe deutscher Musik, 1. Reihe: Reichsdenkmale, 29 Bde., wechselnde Verlagsorte, 1935-43, als Hauptreihe 1956ff. 2. Reihe: Landschaftsdenkmale, 16 Bde.,wechselnde Verlagsorte, 1936-42, als Sonderreihe Kassel bzw. Mainz $1954 \mathrm{ff}$.

Hob (Hoboken-Verz.) Anton van Hoboken, Joseph Haydn. Thematisch-bibliographisches WV, 3 Bde., Mainz 1957-78

HWV (Händel-WV) Bernd Baselt, HändelHandbuch, Bd. 1-3, Thematisch-systematisches Verz., Lpz. 1978
JAMS Journal of the American Musicological Society, 1 (1948) ff.

JRMA Journal of the Royal Music Association, 1 (1986) ff.

K (Kirkpatrick-Verz.) Ralph Kirkpatrick, Domenico Scarlatti, Princeton 1953, ${ }^{3} 1982$, Anhang

KDG Komponisten der Gegenwart, herausgegeben von Hanns-Werner Heister und Walter-Wolfgang Sparrer, München $1992 \mathrm{ff}$.

KV (Köchel-Verzeichnis) Ludwig Ritter von Köchel, chronologisch-thematisches Verzeichnis sämmtlicher Tonwerke W. A. Mozarts, Lpz.1862, 6. Auflage bearbeitet von Franz. Giegling, Alexander Weinmann und Gerd Sievers, Wiesbaden 1969

MB Musica Britannica, Ldn. 1951ff.

MD Musica Disciplina 2 (1948) ff.; (1 unter dem Titel Journal of Renaissance and Baroque Music)

MGG Die Musik in Geschichte und Gegenwart, neubearbeitete, zweite Auflage hrsg. von Ludwig Finscher, Kassel und Stuttgart 1994ff.

MK Musik-Konzepte, hrsg. von Heinz-Klaus Metzger und Rainer Riehn, Mn. 1977ff.

MQ Musical Quarterly, 1 (1915) ff.

NGro- The New Grove Dictionary of Music veDN and Musicians, Second edition edited by Stanley Sadie (u. a.) Ldn. 2001

NZfM Neue Zeitschrift für Musik, 1 (1834) ff.

PFMC Polyphonic Music of the Fourteenth Century, Monaco 1974ff.

PNM Perspectives of New Music, 1 (1962-63) ff.

SMZ Schweizerische Musikzeitung 1 (1861) 123 (1983)

SWV (Schütz-WV), kleine Ausg. hrsg. von Werner Bittinger, Kassel 1960

TVNM Tijdschrift van de Vereeniging voor Nederlandse Muziekgeschiedenis, 1882ff. 


\section{Abkürzungsverzeichnis der amerikanischen Bundesstaaten}

$\begin{array}{ll}\text { AL } & \text { Alabama } \\ \text { AK } & \text { Alaska } \\ \text { AZ } & \text { Arizona } \\ \text { AR } & \text { Arkansas } \\ \text { CA } & \text { California } \\ \text { CO } & \text { Colorado } \\ \text { CT } & \text { Connecticut } \\ \text { DE } & \text { Delaware } \\ \text { DC } & \text { Dist. of Columbia } \\ \text { FL } & \text { Florida } \\ \text { GA } & \text { Georgia } \\ \text { GU } & \text { Guam } \\ \text { HI } & \text { Hawaii } \\ \text { ID } & \text { Idaho } \\ \text { IL } & \text { Illinois } \\ \text { IN } & \text { Indiana } \\ \text { IA } & \text { Iowa } \\ \text { KS } & \text { Kansas } \\ \text { KY } & \text { Kentucky } \\ \text { LA } & \text { Louisiana } \\ \text { ME } & \text { Maine } \\ \text { MD } & \text { Maryland } \\ \text { MA } & \text { Massachusetts } \\ \text { MI } & \text { Michigan } \\ \text { MN } & \text { Minnesota } \\ \text { MS } & \text { Mississippi } \\ \text { MO } & \text { Missouri } \\ & \end{array}$

$\begin{array}{ll}\text { MT } & \text { Montana } \\ \text { NE } & \text { Nebraska } \\ \text { NV } & \text { Nevada } \\ \text { NH } & \text { New Hampshire } \\ \text { NI } & \text { New Jersey } \\ \text { NM } & \text { New Mexico } \\ \text { NY } & \text { New York } \\ \text { NC } & \text { North Carolina } \\ \text { ND } & \text { North Dakota } \\ \text { OH } & \text { Ohio } \\ \text { OK } & \text { Oklahoma } \\ \text { OR } & \text { Oregon } \\ \text { PA } & \text { Pennsylvania } \\ \text { PR } & \text { Puerto Rico } \\ \text { RI } & \text { Rhode Island } \\ \text { SC } & \text { South Carolina } \\ \text { SD } & \text { South Dakota } \\ \text { TN } & \text { Tennessee } \\ \text { TX } & \text { Texas } \\ \text { UT } & \text { Utah } \\ \text { VT } & \text { Vermont } \\ \text { VA } & \text { Virginia } \\ \text { VI } & \text { Virgin Islands } \\ \text { WA } & \text { Washington } \\ \text { WV } & \text { West Virginia } \\ \text { WI } & \text { Wisconsin } \\ \text { WY } & \text { Wyoming }\end{array}$

